\title{
Estado de la urología reconstructiva en Colombia: Tratamiento de la estrechez uretral anterior, una encuesta nacional
}

\section{Status of Reconstructive urology in Colombia: Treatment of Anterior Urethral Stricture, A National Survey}

\author{
Germán A. Patiño ${ }^{1,2}$ Gabriel L. Carreño ${ }^{2}$ Juan Guillermo Prada Gwinner ${ }^{1,2(0)}$ Jaime Perez ${ }^{1,2}$ \\ ${ }^{1}$ Hospital Universitario San Ignacio, Bogotá, Cundinamarca, \\ Address for correspondence Juan Guillermo Prada Gwinner, Hospital \\ Colombia \\ 2 Pontificia Universidad Javeriana, Bogotá, Colombia \\ Universitario San Ignacio, Bogotá, Cundinamarca, Colombia \\ (e-mail: jgpgbarbaro@gmail.com).
}

Urol Colomb 2021;30:5-14.

\section{Resumen}

Palabras clave

- uretra

- uretrotomía

- dilatación uretral

- estrechez uretral

- uretroplastia

- injerto
Purpose El tratamiento mínimamente invasivo de la estrechez uretral tiene altas tasas de recurrencia y re-operación a largo plazo, no obstante, encuestas realizadas en otros países han demostrado que los urólogos tienen poca experiencia con la uretroplastia abierta y hay una preferencia a la utilización de las terapias endoscópicas mínimamente invasivas. El objetivo de este estudio, es describir patrones de práctica del tratamiento de la estrechez de uretra anterior en nuestro país.

Métodos Se trata de un estudio observacional descriptivo y para ello se realizó un cuestionario adaptado a nuestro contexto nacional, basado en varios estudios previamente realizados acerca de la experiencia en Urología reconstructiva. Ese cuestionario incluía información sobre la edad, nivel de experiencia en urología general, la experiencia en urología reconstructiva, escenario de la práctica y las técnicas preferidas para el manejo de las estrecheces uretrales pendulares y bulbares. La información fue almacenada de forma anónima, los datos fueron analizados mediante el paquete estadístico SPSS y se realizó un análisis de distribución de frecuencias.

Resultados Se obtuvieron 106 respuestas de los urólogos encuestados. Para el tratamiento de la estrechez uretral pendular la mayoría de los urólogos prefiere el manejo endoscópico mínimamente invasivo, seguido de uretroplastia con injerto con porcentajes de $69,9 \%$ y $25,5 \%$ respectivamente. Solo el $5 \%$ prefiere derivar a sus pacientes a un centro especializado. Para la estrechez de la uretra bulbar se prefiere las técnicas mínimamente invasivas, uretroplastia y remisión a un centro especializado en un $44,3 \%, 41,5 \%$ y $14,2 \%$ respectivamente. La población más joven y con formación urológica más reciente tiende a hacer más a menudo la uretroplastia con injerto y menos manejo endoscópico, específicamente la uretrotomía interna. En las ciudades received

January 7, 2020

accepted

May 2, 2020

published online

October 6, 2020
DOI https://doi.org/

$10.1055 / \mathrm{s}-0040-1713379$.

ISSN 0120-789X.

e ISSN 2027-0119.
(C) 2020. Sociedad Colombiana de Urología. All rights reserved.

This is an open access article published by Thieme under the terms of the Creative Commons Attribution-NonDerivative-NonCommercial-License, permitting copying and reproduction so long as the original work is given appropriate credit. Contents may not be used for commercial purposes, or adapted, remixed, transformed or built upon. (https://creativecommons.org/ licenses/by-nc-nd/4.0/)

Thieme Revinter Publicações Ltda., Rua do Matoso 170, Rio de Janeiro, RJ, CEP 20270-135, Brazil 


\begin{abstract}
Keywords

- urethra

- urethrotomy

- urethral dilation

- urethral stricture urethroplasty

- graft
\end{abstract}

intermedias, hay una predilección por el tratamiento endoscópico, especialmente uretrotomía interna.

Conclusiones El enfoque de tratamiento mínimamente invasivo de la estrechez uretral es el más frecuentemente elegido a pesar de sus pobres tasas de éxito a largo plazo. Es de destacar que las nuevas generaciones de urólogos muestran más interés y dominio de las técnicas abiertas, tratamiento estándar hoy en día y con bajas tasas de recaídas y reoperación a largo plazo.

The minimally invasive treatment of urethral stricture has higher recurrence and longterm re operation rates. Surveys in other countries have shown that urologists have little experience with open urethroplasty with a preference to the utilization of minimally invasive therapies. We applied a survey to obtain data about practice patterns of the anterior urethral stricture treatment in our country.

Methods A survey was performed with adapted questions to our national context based on several surveys previously conducted in other countries about experience in Reconstructive Urology. This questionnaire included information about age, experience level in general urology, experience in reconstructive urology, scenario of practice, and the preferred techniques handling the pendular and bulbar urethral strictures. All information was collected anonymous and data were analyzed using the statistical software package SPSS, and a frequency distribution analysis was performed. Results A total of 106 answers from respondent urologists were obtained. For the treatment of pendular urethral strictures the vast majority of respondents prefer the minimally invasive endoscopic treatment, following by graft urethroplasty with percentages of $69.9 \%$ and $25.5 \%$ respectively. Only $5 \%$ prefer to refer their patients for care at a specialized center. Regarding the bulbar urethral the urologists prefers to perform minimally invasive management, followed by urethroplasty and refer to a specialized center in percentages of $44.3 \%, 41.5 \%$ and $14.2 \%$ respectively. The younger and more recent urological trained population tends to make more often graft urethroplasty and less endoscopic management, specifically internal urethrotomy. In the intermediate cities there is a predilection for endoscopic treatment especially internal urethrotomy.

Conclusions The minimally invasive treatment approach of urethral stricture is the most frequently chosen despite its poor long-term success rates. It is noteworthy that the new generations of urologists show more interest and mastery of open techniques, which today is the standard treatment with low relapse and long-term re operation rates.

\section{Introducción}

La estrechez uretral es común y recurrente en la práctica actual de la urología. Se ha considerado que es más común en países en desarrollo ${ }^{1}$ y su etiología cambia de acuerdo con la distribución geográfica. $^{2}$ La causa más común en países desarrollados es iatrogénica y ocurre luego de la cateterización uretral y procedimientos endoscópicos del tracto urinario inferior que causan trauma, fibrosis y cicatrización del lumen uretral. ${ }^{3}$

El tratamiento ideal para la estrechez uretral debe ser seguro, efectivo y duradero con mínimos efectos adversos. Se han propuesto varios enfoques de tratamiento: La dilatación simple ciega ha sido practicada durante muchos siglos y fue la primera línea de manejo para el manejo de estrechez uretral, ${ }^{4}$ posteriormente, la primera uretroplastia abierta se describió en 1945, sin embargo, no se popularizó ampliamente debido a la aparición de técnicas menos dispendiosas y mínimamente invasivas como la uretrotomía interna óptica la cual apareció en 1973 utilizando un endoscopio. ${ }^{5}$

La dilatación simple genera laceraciones sobre la cicatriz anular de la estrechez en varios puntos. El argumento detrás de esa práctica es que esas pequeñas disrupciones cicatrizan con el epitelio sano. La uretrotomía por otro lado, resulta en una incisión única y controlada por lo que se espera que la cicatrización sea mejor y no empeore la estrechez. A pesar de esas hipótesis, múltiples estudios han demostrado que los resultados a largo plazo de las técnicas mínimamente invasivas son ineficaces y generan empeoramiento de la cicatrización. ${ }^{6-8}$ Esa práctica se ha convertido en el estándar en los países que tienen acceso a equipos endoscópicos, y por lo menos el 50\% de los pacientes desarrollarán una estrechez recurrente requiriendo procedimientos múltiples durante su vida. ${ }^{9}$ 
La uretroplastia es una técnica abierta en la cual se identifica el sitio de la estrechez y se reconstruye la uretra ya sea mediante la escisión del segmento enfermo y la anastomosis de los dos segmentos sanos, o incidiendo longitudinalmente la sección obliterada e incorporando material de injerto para mantener el lumen abierto. ${ }^{10}$ La uretroplastia de escisión y anastomosis tiene una alta tasa de éxito, ${ }^{11}$ es más recomendable para estrecheces bulbares cortas $(1 \mathrm{a} 3 \mathrm{~cm}$ ) y se asocia a un éxito de entre 5 y 10 años, del $93 \%{ }^{12,13}$ Esa técnica también ha demostrado ser costo efectiva en longitudes de $2 \mathrm{~cm}$, en estudios de modelos económicos. ${ }^{14,15}$ No obstante, la dilatación uretral y la uretrotomía interna siguen siendo de lejos los tratamientos más comúnmente practicados, probablemente debido a su simplicidad, facilidad de aprendizaje y repetición, y falta de familiaridad con la uretroplastia abierta ${ }^{6,7,10}$ a pesar de una alta tasa de recurrencia predecible. ${ }^{16}$

Encuestas realizadas en varios países a urólogos han mostrado datos interesantes en cuanto a la elección del tratamiento de esa entidad. En Estados Unidos y Holanda se observó que la mayoría de los urólogos tiene poca experiencia con la uretroplastia y a pesar de la predicción en la falla al tratamiento con las terapias mínimamente invasivas, ellas se siguen realizando muy frecuentemente y de forma repetida. ${ }^{17,18}$

En múltiples encuestas realizadas a nivel mundial, se ha encontrado que el manejo de la patología depende mayormente de la experiencia en el manejo de la patología uretral y la familiarización con las diferentes técnicas y no por las indicaciones descritas en las diferentes guías de manejo. ${ }^{16-20}$ Hasta donde tenemos conocimiento, no existen datos en la población colombiana acerca de las preferencias e inclinaciones en cuanto al manejo de la estrechez uretral anterior. Por lo que el objetivo de este trabajo es describir las técnicas que actualmente se prefieren para el manejo de la patología uretral y observar si existe alguna relación con el nivel de experiencia urológica en general y la experiencia específicamente en urología reconstructiva.

\section{Métodos}

Se realizó un estudio observacional descriptivo, posterior a la aprobación del comité de ética de nuestra institución, se diseñó una encuesta dirigida a todos los urólogos de Colombia miembros de la sociedad colombiana de Urología (SCU), a través del correo electrónico. El cuestionario tuvo 8 preguntas y se basó en varios sondeos realizados previamente en otros países adaptando las preguntas al contexto nacional. El cuestionario incluye preguntas acerca de la edad, el nivel de experiencia en urología general, el nivel de experiencia en urología reconstructiva, el escenario de práctica (ciudad intermedia o ciudad capital), y las técnicas preferidas en el manejo de la estrechez de uretra pendular y uretra bulbar (Anexo 3).

Los resultados se recibieron por correo electrónico y todos los datos obtenidos se recopilaron y organizaron en una base de datos utilizando el programa Excel de Microsoft Office®.

Se incluyeron todos los urólogos y residentes miembros de la SCU. La sociedad colombiana de urología afilia a todos los urólogos graduados y a los residentes que se encuentren cursando por lo menos el segundo año de especialización. Se excluyeron los miembros que no ejerzan la profesión en el territorio nacional y/o que no sean miembros activos de la SCU en el momento de realizar la encuesta.

Los datos se analizaron utilizando el paquete de software estadístico IBM SPSS versión $20 \circledR$, y se realizó un análisis por distribución de frecuencias y un análisis de subgrupos. Debido a que es una encuesta anónima y sin tratamiento de información de pacientes, no fue necesaria autorización del Comité de Investigaciones de la Institución.

\section{Resultados}

De los 550 miembros de la sociedad colombiana de urología, se obtuvo respuesta de 106 urólogos quienes contestaron la encuesta. La - Tabla 1 resume los hallazgos generales de la encuesta.

La mayoría de los urólogos se encontraba entre los 36 y los 50 años, seguido de urólogos entre 25 y 35 años y en menor

Tabla 1 Resumen de la información general obtenida de la encuesta

\begin{tabular}{|c|c|c|}
\hline & & No. (\%) \\
\hline \multirow[t]{4}{*}{ Edad } & $25-35$ & $26(24.5)$ \\
\hline & $36-50$ & $47(44.3)$ \\
\hline & $51-60$ & $21(19.8)$ \\
\hline & Mayor de 60 & $12(11.3)$ \\
\hline \multirow[t]{4}{*}{ Años de práctica } & Menos de 5 & $29(27.4)$ \\
\hline & $5-10$ & $16(15.1)$ \\
\hline & $11-20$ & $33(31.1)$ \\
\hline & Más de 20 & $28(26.4)$ \\
\hline \multirow[t]{2}{*}{ Lugar de la práctica } & Ciudad capital & $84(79.2)$ \\
\hline & Ciudad Intermedia & $22(20.8)$ \\
\hline \multirow{2}{*}{$\begin{array}{l}\text { Maneja pacientes } \\
\text { con estrechez } \\
\text { uretral }\end{array}$} & Sí & $98(92.5)$ \\
\hline & No & $8(7.5)$ \\
\hline \multirow{4}{*}{$\begin{array}{l}\text { Cantidad de } \\
\text { pacientes }\end{array}$} & $1-5$ & $80(75.5)$ \\
\hline & $6-10$ & 20 (18.9) \\
\hline & $11-20$ & $2(1.9)$ \\
\hline & Más de 20 & $4(3.8)$ \\
\hline \multirow{4}{*}{$\begin{array}{l}\text { Manejo de la } \\
\text { estrechez } \\
\text { de uretra pendular }\end{array}$} & Cistoscopia y dilatación & $45(42.5)$ \\
\hline & Uretrotomía interna & $29(27.4)$ \\
\hline & Uretroplastia con injerto & $27(25.5)$ \\
\hline & $\begin{array}{l}\text { Remisión a centro } \\
\text { especializado }\end{array}$ & $5(4.7)$ \\
\hline \multirow{4}{*}{$\begin{array}{l}\text { Manejo de la } \\
\text { estrechez } \\
\text { de uretra bulbar }\end{array}$} & Uretrotomía interna & $47(44.3)$ \\
\hline & Uretroplastia perineal & 37 (34.9) \\
\hline & Uretroplastia con injerto & $7(6.6)$ \\
\hline & $\begin{array}{l}\text { Remisión a centro } \\
\text { especializado }\end{array}$ & $15(14.2)$ \\
\hline \multirow{2}{*}{$\begin{array}{l}\text { Interés en foros de } \\
\text { urología } \\
\text { reconstuctiva }\end{array}$} & Sí & $96(90.6)$ \\
\hline & No & $10(9.4)$ \\
\hline
\end{tabular}


proporción de 51 a 60 años con porcentajes de $44,3 \%, 24,5 \%$, y $19,8 \%$ respectivamente. Con respecto a la experiencia en la práctica de la urología, el 42,5\% tenía entre 0 y 10 años y el 57,5\% tenía 10 o más años de experiencia.

La gran mayoría de los encuestados desempeña sus labores en una ciudad capital (79,2\%), y casi todos atienden pacientes con patología obstructiva de la uretra. El 75\% trata entre 1 a 5 pacientes con estrechez uretral al mes, y solo una minoría trata un volumen superior a los 20 pacientes mensuales.

La mayoría de los profesionales que realizan intervenciones en pacientes con patología uretral, se encuentran entre 36 y 50 años, de los cuales más de la mitad viven en una ciudad capital, y tienen más de 10 años de experiencia en el campo de la Urología.
La población más joven y con formación urológica más reciente, tiende a realizar a menudo la uretroplastia con injerto y menos frecuentemente el manejo endoscópico específicamente con uretrotomía interna comparado con la población, además, destaca que la gran mayoría tiene interés en el campo de la urología reconstructiva y desea que se realicen más jornadas académicas en cuanto al manejo de la patología obstructiva uretral. El tratamiento de la patología uretral es similar en los grupos de edades mayores a 50 años prefiriendo realizar tratamiento endoscópico como primera opción (-Tabla 2).

Los Urólogos que remiten pacientes a centros especializados en tratamiento de la patología uretral son aquellos que evalúan la menor cantidad de pacientes al mes,

Tabla 2 Tabla de resultados del análisis en cuanto a la distribución por edad en años

\begin{tabular}{|c|c|c|c|c|}
\hline \multirow[t]{2}{*}{ Variables } & \multicolumn{4}{|c|}{ Distribución por edad (en años) } \\
\hline & 25-35 No. (\%) & 36-50 No. (\%) & 51-60 No. (\%) & Más de 60 No. (\%) \\
\hline \multicolumn{5}{|l|}{ Años de práctica } \\
\hline Menos de 5 & $24(92.3)$ & $5(10.6)$ & $0(0)$ & $0(0)$ \\
\hline $5-10$ & $2(7.7)$ & $14(29.8)$ & $0(0)$ & $0(0)$ \\
\hline $11-20$ & $0(0)$ & $26(55.3)$ & $6(28.6)$ & $1(8.3)$ \\
\hline Más de 20 & $0(0)$ & $2(4.3)$ & $15(71.4)$ & $11(91.7)$ \\
\hline Total & $26(100)$ & $47(100)$ & $21(100)$ & $12(100)$ \\
\hline \multicolumn{5}{|l|}{ Lugar } \\
\hline Ciudad capital & $23(88.5)$ & $34(72.3)$ & $18(85.7)$ & $9(75)$ \\
\hline Ciudad intermedia & $3(11.5)$ & $13(27.7)$ & $3(14.3)$ & $3(25)$ \\
\hline Total & $26(100)$ & $47(100)$ & $21(100)$ & $12(100)$ \\
\hline \multicolumn{5}{|l|}{ Cantidad de pacientes } \\
\hline $1-5$ & $18(69.2)$ & $39(83)$ & $15(71.4)$ & $8(66.7)$ \\
\hline $6-10$ & 7 (26.9) & $5(10.6)$ & $6(28.6)$ & $2(16.7)$ \\
\hline $11-20$ & $0(0)$ & $2(4.3)$ & $0(0)$ & $0(0)$ \\
\hline Más de 20 & $1(3.8)$ & $1(2.1)$ & $0(0)$ & $2(16.7)$ \\
\hline Total & $26(100)$ & $47(100)$ & $21(100)$ & $12(100)$ \\
\hline \multicolumn{5}{|l|}{ Uretra anterior } \\
\hline $\begin{array}{l}\text { Cistoscopia y } \\
\text { dilatación }\end{array}$ & $13(50)$ & $18(38.3)$ & $8(38.1)$ & $6(50)$ \\
\hline Uretrotomía interna & $4(15.4)$ & $12(25.5)$ & $7(33.3)$ & $6(50)$ \\
\hline Uretroplastia con injerto & $9(34.6)$ & $13(27.7)$ & $5(23.8)$ & $0(0)$ \\
\hline $\begin{array}{l}\text { Remisión a } \\
\text { Centro especializado }\end{array}$ & $0(0)$ & $4(8.5)$ & $1(4.8)$ & $0(0)$ \\
\hline Total & $26(100)$ & $47(100)$ & $21(100)$ & $12(100)$ \\
\hline \multicolumn{5}{|l|}{ Uretra bulbar } \\
\hline Uretrotomía interna & $15(57.7)$ & $20(42.6)$ & $8(38.1)$ & $4(33.3)$ \\
\hline Uretroplastia perineal & $8(30.8)$ & $13(27.7)$ & $10(47.6)$ & $6(50)$ \\
\hline Uretroplastia con injerto & $1(3.8)$ & $5(10.6)$ & $0(0)$ & $1(8.3)$ \\
\hline Remisión a centro especializadoa & $2(7.7)$ & $9(19.1)$ & $3(14.3)$ & $1(8.3)$ \\
\hline Total & $26(100)$ & $47(100)$ & $21(100)$ & $12(100)$ \\
\hline
\end{tabular}


sin embargo, la proporción de pacientes enviados a centros especializados es baja.

Al dividir la población de Urólogos en aquellos que trabajan en una ciudad capital y los que laboran en otras ciudades, los resultados muestran que en promedio, la gran mayoría de los urólogos del país bien sea de ciudad capital o intermedia, no evalúan un número mayor a 10 pacientes al mes. En el rango de edad menor a 50 años, se encuentran los que más uretroplastias realizan, en cambio el tratamiento endoscópico es la primera elección en urólogos mayores de 50 años (-Tabla 3).

Tabla 3 Información de acuerdo al desempeño en ciudad capital versus Ciudad intermedia

\begin{tabular}{|c|c|c|}
\hline & $\begin{array}{l}\text { Ciudad } \\
\text { capital }\end{array}$ & $\begin{array}{l}\text { Ciudad } \\
\text { intermedia No. }\end{array}$ \\
\hline & No. (\%) & (\%) \\
\hline Edad en años & & \\
\hline $25-35$ & $23(27.4)$ & $3(13.6)$ \\
\hline $36-50$ & $34(40.5)$ & $13(59.1)$ \\
\hline $51-60$ & $18(21.4)$ & $3(13.6)$ \\
\hline Más de 60 & $9(10.7)$ & $3(13.6)$ \\
\hline Total & $84(100)$ & $22(100)$ \\
\hline Años de práctica & & \\
\hline Menos de 5 & $26(31)$ & $3(13.6)$ \\
\hline $5-10$ & $9(10.7)$ & $7(31.8)$ \\
\hline $11-20$ & $27(32.1)$ & $6(27.3)$ \\
\hline Más de 20 & $22(26.2)$ & $6(27.3)$ \\
\hline Total & $84(100)$ & $22(100)$ \\
\hline Cantidad de pacientes & & \\
\hline $1-5$ & $63(75)$ & $17(77.3)$ \\
\hline $6-10$ & 16 (19) & $4(18.2)$ \\
\hline $11-20$ & $2(2.4)$ & $0(0)$ \\
\hline Más de 20 & $3(3.6)$ & $1(4.5)$ \\
\hline Total & $84(100)$ & $22(100)$ \\
\hline Uretra pendular & & \\
\hline Cistoscopia y dilatación & $37(44)$ & $8(36.4)$ \\
\hline Uretrotomía interna & $19(22.6)$ & $10(45.5)$ \\
\hline Uretroplastia con injerto & $24(28.6)$ & $3(13.6)$ \\
\hline $\begin{array}{l}\text { Remisión a centro } \\
\text { especializado }\end{array}$ & $4(4.8)$ & $1(4.5)$ \\
\hline Total & $84(100)$ & $22(100)$ \\
\hline Uretra bulbar & & \\
\hline Uretrotomía interna & $39(46.4)$ & $8(36.4)$ \\
\hline Uretroplastia perineal & $30(35.7)$ & $7(31.8)$ \\
\hline Uretroplastia con injerto & $4(4.8)$ & $3(13.6)$ \\
\hline $\begin{array}{l}\text { Remisión a centro } \\
\text { especializado }\end{array}$ & $11(13.1)$ & $4(18.2)$ \\
\hline Total & $84(100)$ & $22(100)$ \\
\hline
\end{tabular}

De acuerdo con el volumen de pacientes que maneja cada subgrupo de urólogos al mes, es notable la predilección por realizar más uretroplastia con injerto a medida que aumenta el volumen de pacientes, pero se observa concomitantemente un aumento en el porcentaje de los que practican técnicas mínimamente invasivas, observando que en el grupo que atiende de 11-20 pacientes al mes, donde el 50\% realiza uretrotomía interna y nadie realiza cistoscopia y dilatación, mientras que en el subgrupo de los que evalúan más de 20 al mes, ninguno realiza uretrotomías y el 50\% prefiere la dilatación bajo cistoscopia ( - Tabla 4 ).

\section{Uretra Pendular}

En términos generales la gran mayoría de los encuestados prefiere el manejo endoscópico mínimamente invasivo para la estrechez de uretra pendular (69,9\%), y el $27,4 \%$ prefiere la uretrotomía interna como primera elección. Una cuarta parte practica la uretroplastia con injerto $(25,5 \%)$ y solo el $5 \%$ prefiere remitir sus pacientes para atención en un centro especializado. Se observa una relación directamente proporcional a la edad y la experiencia de realizar más uretrotomía interna para el manejo de la estrechez de uretra anterior. Es evidente que el manejo de la uretra pendular es más frecuentemente endoscópico en las ciudades intermedias y mayormente mediante uretrotomía. Aunque en las ciudades capitales también es más frecuente el manejo endoscópico, al compararlo con las ciudades intermedias es menor y el porcentaje del uso de la técnica con injerto es el doble que en las ciudades intermedias ( $28,6 \%$ vs $13,6 \%$ ). (ver - Tabla 3.)

\section{Uretra Bulbar}

Acerca del manejo de la estrechez de uretra bulbar, el escenario es similar en cuanto a que casi la mitad opta por el manejo endoscópico con uretrotomía interna óptica (44,3\%). Un porcentaje importante de los encuestados realiza uretroplastia perineal $(34,9 \%)$ y en menor proporción se prefiere una técnica con injerto (6,6\%). El manejo de la estrechez de uretra bulbar, demuestra una relación inversamente proporcional entre la edad del urólogo y la realización de uretrotomía interna, mientras que a medida que aumenta la edad y la experiencia se opta mayormente por la uretroplastia perineal. Igualmente, una minoría realiza uretroplastia con injerto y el porcentaje de remisión a centros especializados es similar.

\section{Discusión}

La estrechez uretral es una causa común de obstrucción del tracto urinario inferior a través de toda la historia. Las técnicas abiertas para el tratamiento de esa condición son altamente efectivas y duraderas, pero son invasivas y requieren cateterización prolongada y habilidades quirúrgicas especializadas. No es de asombrarnos de que exista una inclinación mundial a realizar tratamientos mínimamente invasivos para el manejo de la estrechez uretral sin embargo, a diferencia de otras técnicas 
Tabla 4 Información de acuerdo a subgrupos de volumen de pacientes tratados al mes

\begin{tabular}{|c|c|c|c|c|}
\hline \multirow[t]{2}{*}{ Variables } & \multicolumn{4}{|c|}{ Volumen de pacientes al mes } \\
\hline & 1 - 5 No. (\%) & $6-10$ No. (\%) & $11-20$ No. (\%) & Más de 20 No. (\%) \\
\hline \multicolumn{5}{|l|}{ Edad en años } \\
\hline $25-35$ & $18(22.5)$ & $7(35)$ & $0(0)$ & $1(25)$ \\
\hline $36-50$ & $39(48.8)$ & $5(25)$ & $2(100)$ & $1(25)$ \\
\hline $51-60$ & $15(18.8)$ & $6(30)$ & $0(0)$ & $0(0)$ \\
\hline Más de 60 & $8(10)$ & $2(10)$ & $0(0)$ & $2(50)$ \\
\hline Total & $80(100)$ & $20(100)$ & $2(100)$ & $4(100)$ \\
\hline \multicolumn{5}{|l|}{ Años de práctica } \\
\hline Menos de 5 & $20(25)$ & $7(35)$ & $1(50)$ & $1(25)$ \\
\hline $5-10$ & $15(18.8)$ & $1(5)$ & $0(0)$ & $0(0)$ \\
\hline $11-20$ & $26(32.5)$ & $4(20)$ & $1(50)$ & $2(50)$ \\
\hline Más de 20 & $19(23.8)$ & $8(40)$ & $0(0)$ & $1(25)$ \\
\hline Total & $80(100)$ & $20(100)$ & $2(100)$ & $4(100)$ \\
\hline \multicolumn{5}{|l|}{ Lugar } \\
\hline Ciudad capital & $63(78.8)$ & $16(80)$ & $2(100)$ & $3(75)$ \\
\hline Ciudad intermedia & $17(21.3)$ & $4(20)$ & $0(0)$ & $1(25)$ \\
\hline Total & $80(100)$ & $20(100)$ & $2(100)$ & $4(100)$ \\
\hline \multicolumn{5}{|l|}{ Uretra pendular } \\
\hline Cistoscopia y & \multirow[t]{2}{*}{$37(46.3)$} & \multirow[t]{2}{*}{$6(30)$} & \multirow[t]{2}{*}{$1(50)$} & \multirow[t]{2}{*}{$2(50)$} \\
\hline dilatación & & & & \\
\hline Uretrotomía interna & $20(25)$ & $8(40)$ & $1(50)$ & $0(0)$ \\
\hline Uretroplastia con injerto & $18(22.5)$ & $6(30)$ & $0(0)$ & $2(50)$ \\
\hline Remisión a centro especializado & $5(6.3)$ & $0(0)$ & $0(0)$ & $0(0)$ \\
\hline Total & $80(100)$ & $20(100)$ & $2(100)$ & $4(100)$ \\
\hline \multicolumn{5}{|l|}{ Uretra bulbar } \\
\hline Uretrotomía interna & $38(47.5)$ & $8(40)$ & $1(50)$ & $0(0)$ \\
\hline Uretroplastia perineal & $24(30)$ & $10(50)$ & $1(50)$ & $2(50)$ \\
\hline Uretroplastia con injerto & $5(6.3)$ & $1(5)$ & $0(0)$ & $1(25)$ \\
\hline Remisión a centro especializado & $13(16.3)$ & $1(5)$ & $0(0)$ & $1(25)$ \\
\hline Total & $80(100)$ & $20(100)$ & $2(100)$ & $4(100)$ \\
\hline
\end{tabular}

quirúrgicas para el tratamiento de patologías urológicas, la técnica endoscópica en el manejo de la estrechez uretral no ha demostrado ser exitosa a pesar de su amplio uso y reproducibilidad. ${ }^{20}$ Andrich y col., sugieren que la uretrotomía y la dilatación uretral son excesivamente e inapropiadamente utilizadas debido a su simplicidad, reproducibilidad y falta de familiarización con técnicas de cirugía abierta. ${ }^{21}$ Nuestros resultados demuestran que en general el manejo de la patología uretral en Colombia sigue el mismo patrón global actual. ${ }^{16-20}$ Aproximadamente un $70 \%$ realiza manejo endoscópico de primera línea en estrecheces pendulares, lo cual llama la atención dado que no se recomienda realizar ese tipo de intervención por la alta tasa de recaída. Además, se realiza uretrotomía interna en el $50 \%$ de los casos de estrechez de uretra bulbar lo cual concuerda con lo descrito en otras publicaciones. ${ }^{16-20}$
Bullock y col., demuestran en su encuesta nacional en los Estados Unidos que el 74\% de los Urólogos encuestados erróneamente cree que el manejo de la estrechez uretral es una escalera que inicia con procedimientos endoscópicos, mientras que el manejo abierto definitivo solo se contempla luego de sucesivas fallas. Asimismo, existe una creencia equivocada que la estrechez se estabiliza luego de múltiples uretrotomías lo que sucede por la falta de familiaridad y poca experiencia en la uretroplastia abierta. ${ }^{17}$ Pansadoro y col., reportaron en su serie de 224 pacientes tratados con uretrotomía interna óptica con un seguimiento a 5 años, que la recurrencia luego del primer procedimiento era del $68 \%$ y del 96\% después del segundo. ${ }^{22}$ Heyns y col., en un estudio aleatorizado prospectivo, encontraron resultados similares con recurrencia en un seguimiento de 48 meses en el umbral del $61 \%$ y $100 \%$ después de la primera y segunda 


\section{Uretra pendular}

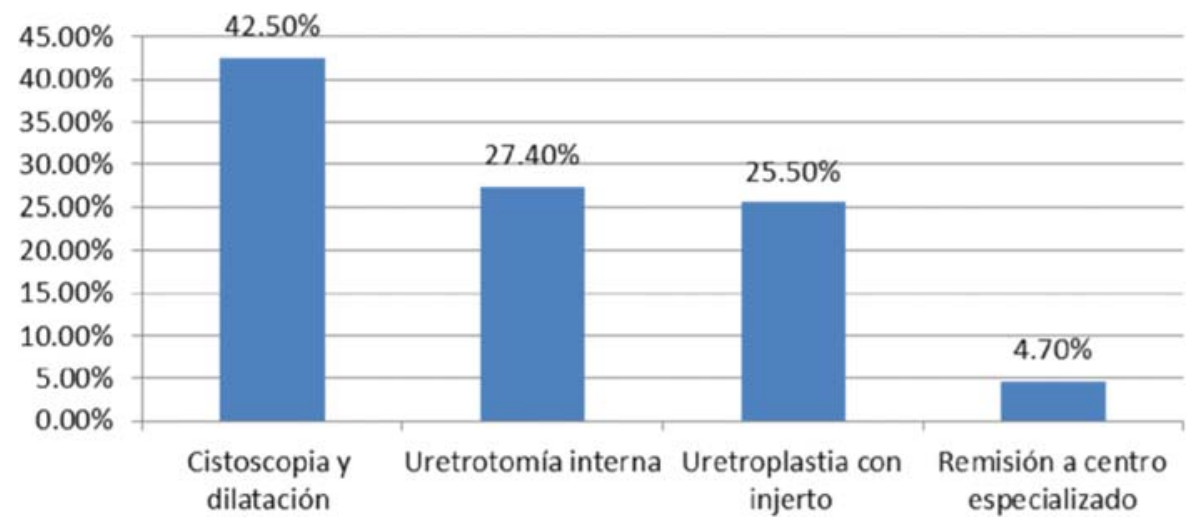

Fig. 1 Resumen de los tratamientos de elección para el tratamiento de la estrechez uretral pendular.

\section{Uretra bulbar}

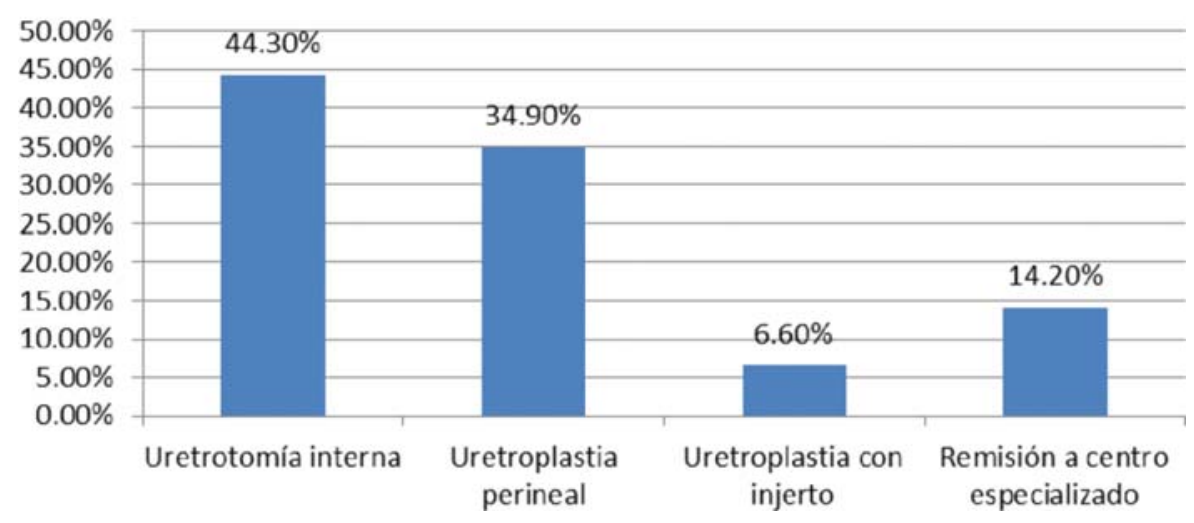

Fig. 2 Resumen de los tratamientos de elección para el tratamiento de la estrechez uretral bulbar.

\section{Cantidad de pacientes al mes}

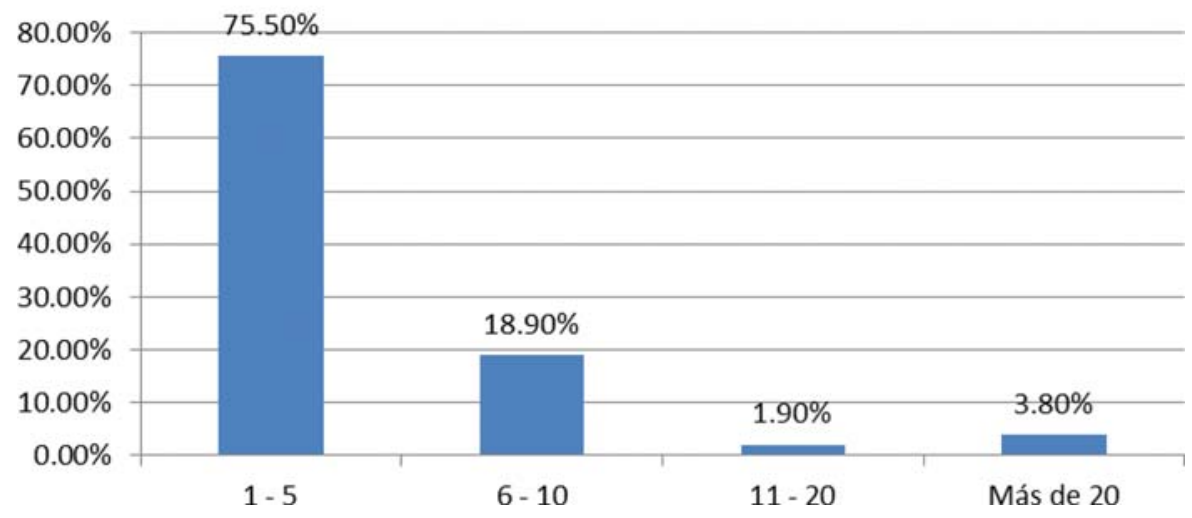

Fig. 3 Resumen del porcentaje de urólogos de acuerdo al volumen de pacientes que tratan mensualmente con estrechez uretral.

uretrotomía respectivamente. ${ }^{6}$ Santucci y col., realizaron una revisión retrospectiva y reportaron una recurrencia del $92 \%$, $94 \%, 91 \%, 100 \%$ y $100 \%$ después de la primera, segunda, tercera, cuarta y quinta uretrotomía y tiempos de recurrencia de 7, 9, 3, 20 y 8 meses respectivamente. ${ }^{23}$

Los cambios recientes en el entrenamiento en urología reconstructiva son evidentes, puesto que al analizar los grupos por edad, encontramos que la mayoría de las uretroplastias con injerto las realizan los urólogos menores de 35 años pues en ese grupo el porcentaje que realiza ese abordaje quirúrgico es el mayor (34,6\%). Van Leeuwen y col., quienes realizaron una encuesta similar en Holanda, describen una necesidad en el entrenamiento ya que que únicamente el $3 \%$ de los urólogos menores de 50 años realizan más de 5 uretroplastias al año. ${ }^{18}$ Uno de los hallazgos destacables de nuestro estudio es que los urólogos mayores de 50 años, 
realizan más frecuentemente uretroplastia perineal para el manejo de la estrechez de uretra bulbar que los urólogos menores de 50 años, técnica ampliamente conocida, aunque dispendiosa, de larga duración y con requerimiento de hospitalización. Andrich y col., describe su experiencia con un programa de entrenamiento de Fellowship en urología reconstructiva y demostró cuánto tiempo y esfuerzo toma adquirir esa habilidad en uretroplastia. ${ }^{19}$ En nuestro trabajo, encontramos que en general la mayoría de los urólogos evalúan un bajo volumen de pacientes con estrechez uretral al mes, lo cual limita la experiencia de los Urólogos en el manejo de estrechez uretral, el 75\% de los encuestados maneja menos de 5 pacientes con estrechez uretral al mes, lo cual limita la experiencia de los urólogos en el manejo de la patología uretral.

Bullock y col., proponen que la popularidad de las técnicas mínimamente invasivas se relaciona con la falta de familiaridad respecto de la técnica de uretroplastia abierta o con la inhabilidad de obtener un número suficiente de pacientes para mantener la habilidad quirúrgica. Ellos reportan que la uretroplastia solo la realizan un $42 \%$ de los encuestados, y de ellos, un $89 \%$ realiza menos de 5 cirugías al año, y solo un $1,2 \%$ lleva a cabo más de $10 .{ }^{17}$ En nuestro estudio, con respecto al manejo de la uretra pendular, es evidente que el manejo endoscópico es el más frecuente en todo el grupo de urólogos encuestados, hallazgo que se correlaciona con lo descrito en la literatura, sin embargo, como habíamos mencionado previamente, ese manejo no es recomendado. ${ }^{24}$

Los injertos de mucosa oral fueron descritos por primera vez en 1941 en una serie de reparo de hipospadias en un tiempo. Desde entonces, y gracias al refinamiento de la técnica, se ha convertido en el procedimiento de elección para estrecheces de gran longitud. En nuestro estudio, el 25\% de los encuestados realizan uretroplastia con injerto. La ventajas atribuibles al injerto de mucosa oral son la facilidad en la obtención, disponibilidad, resistencia a la infección y características del tejido (grosor epitelial, alto contenido de fibras elásticas y lámina propia delgada). ${ }^{25}$

Con respecto al manejo de la estrechez bulbar es notable que la distribución es igualmente proporcional entre el manejo endoscópico y el tratamiento abierto, lo cual es más acorde a las recomendaciones actuales puesto que existe un papel para la uretrotomía interna como intento inicial único para el manejo de estrecheces bulbares menores de $1 \mathrm{~cm}$ de etiología no traumática sin evidencia de espongiofibrosis importante. ${ }^{24}$ La uretroplastia de escisión y anastomosis primaria es más apta para estrecheces de $1-3 \mathrm{~cm}$ con tasas de éxito del $93 \%$ a 5 y 10 años. ${ }^{12,13}$

Una de las limitantes de nuestro trabajo fue la no existencia de una encuesta estandarizada para evaluar el manejo de la estrechez uretral y sus patologías asociadas, existen múltiples a nivel mundial pero no hay ninguna validada. El grupo de investigación desarrolló esa tarea basado en encuestas previas realizadas a nivel mundial, dado lo anterior los datos deben ser analizados con cautela, sin embargo es la primera aproximación al manejo de la patología uretral anterior en nuestro país y recalca las limitaciones que tienen los Urólogos en Colombia para el manejo de patología uretral anterior. Así mismo, otra limitante fue el número de respuestas obtenidas teniendo una base de participantes conocida, pero como está descrito en la literatura, nuestros números están dentro del resultado promedio de las encuestas a nivel mundial, siendo una población suficientemente representativa. Estudios han demostrado que los incentivos mejoran un poco el índice de respuesta. ${ }^{26}$

\section{Conclusiones}

Los urólogos en Colombia prefieren para el manejo de las lesiones uretrales, el abordaje mínimamente invasivo, con un bajo uso de las técnicas abiertas. El desconocimiento de las mismas y la falta de exposición a mayores volúmenes de pacientes con esa patología, hace necesario la creación de centros especializados en reconstrucción, como también la creación de programas de formación. Algunos de los urólogos más jóvenes han tenido una mayor exposición al entrenamiento en uretroplastia, lo cual ha generado un cambio en el enfoque del tratamiento de la estrechez uretral.

\section{Referencias}

1 Brandes SB. Epidemiology, Etiology, Histology, Classification, and Economic Impact of Urethral Stricture Disease. In: Urethral Reconstructive Surgery. Humana Press; 2008:53-61

2 Stein DM, Thum DJ, Barbagli G, et al. A geographic analysis of male urethral stricture aetiology and location. BJU Int 2013;112(06): 830-834

3 Mathur R, Aggarwal G, Satsangi B, Khan F, Odiya S. Comprehensive analysis of etiology on the prognosis of urethral strictures. Int Braz J Urol [Internet] [cited 2019 Dec 17];37(03):362-369; discussion 369-70. Available from: http://www.ncbi.nlm. nih.gov/pubmed/21756384

4 Attwater HL. The history of urethral stricture. BJUI 1943;15(02): 39-51

5 Chiou RK, Taylor RJ. Changing concepts of urethral stricture management. II: Selection of treatment options. Nebr Med J 1996;81(08):287-291http://www.ncbi.nlm.nih.gov/pubmed/ 8810229 [Internet]

6 Heyns CF, Steenkamp JW, De Kock MLS, Whitaker P. Treatment of male urethral strictures: is repeated dilation or internal urethrotomy useful? J Urol 1998;160(02):356-358

7 Steenkamp JW, Heyns CF, de Kock MLS. Internal urethrotomy versus dilation as treatment for male urethral strictures: a prospective, randomized comparison. J Urol 1997;157(01):98-101

8 Roehrborn CG, McConnell JD. Analysis of factors contributing to success or failure of 1-stage urethroplasty for urethral stricture disease. J Urol 1994;151(04):869-874http://www.ncbi.nlm. nih.gov/pubmed/8126813 [Internet]

9 Holm-Nielsen A, Schultz A, Møller-Pedersen V. Direct vision internal urethrotomy. A critical review of 365 operations. Br J Urol 1984;56(03):308-312http://www.ncbi.nlm.nih.gov/pubmed/ 6544616 [Internet]

10 Andrich DE, Mundy AR. What is the best technique for urethroplasty? Eur Urol 2008;54(05):1031-1041

11 Chapple C, Andrich D, Atala A, et al. SIU/ICUD Consultation on Urethral Strictures: The management of anterior urethral stricture disease using substitution urethroplasty. Urology 2014;83(3, Suppl)S31-S47

12 Andrich DE, Dunglison N, Greenwell TJ, Mundy AR. The long-term results of urethroplasty. J Urol 2003;170(01):90-92

13 Micheli E, Ranieri A, Peracchia G, Lembo A. End-to-end urethroplasty: long-term results. BJU Int 2002;90(01):68-71 
14 Wright JL, Wessells $\mathrm{H}$, Nathens AB, Hollingworth W. What is the most cost-effective treatment for 1 to $2-\mathrm{cm}$ bulbar urethral strictures: societal approach using decision analysis. Urology 2006;67(05):889-893

15 Rourke KF, Jordan GH. Primary urethral reconstruction: the cost minimized approach to the bulbous urethral stricture. J Urol 2005;173(04):1206-1210

16 Chilton CP, Shah PJ, Fowler CG, Tiptaft RC, Blandy JP. The impact of optical urethrotomy on the management of urethral strictures. Br J Urol 1983;55(06):705-710http://www.ncbi.nlm.nih.gov/pubmed/ 6652443 [Internet]

17 Bullock TL, Brandes SB. Adult anterior urethral strictures: a national practice patterns survey of board certified urologists in the United States. J Urol 2007;177(02):685-690

18 van Leeuwen MA, Brandenburg JJ, Kok ET, Vijverberg PLM, Bosch JLHR. Management of adult anterior urethral stricture disease: nationwide survey among urologists in the Netherlands. Eur Urol 2011;60(01):159-166

19 Andrich DE, Mundy AR. A Fellowship programme in reconstructive urological surgery: what is it and what is it for? BJU Int 2010;106(01):108-111

20 Barbagli G, Lazzeri M. Surgical treatment of anterior urethral stricture diseases: brief overview. Int Braz J Urol [Internet] [cited
2019 Dec 17];33(04):461-469. Available from: http://www.ncbi. nlm.nih.gov/pubmed/17767749

21 Andrich DE, Greenwell TJ, Mundy AR. Treatment of pelvic fracture-related urethral trauma: a survey of current practice in the UK. BJU Int 2005;96(01):127-130

22 Pansadoro V, Emiliozzi P. Internal urethrotomy in the management of anterior urethral strictures: long-term followup. J Urol 1996;156(01):73-75http://www.ncbi.nlm.nih. gov/pubmed/8648841 [Internet]

23 Santucci R, Eisenberg L. Urethrotomy has a much lower success rate than previously reported. J Urol 2010;183(05):1859-1862

24 Gil FG, Benavides JA, Montoya AD. Guia colombiana para el manejo integral de la estrechez de uretra. Revista Urología Colombiana / Colombian. Urol J 2019;28(03):193-195. Doi: 10.1055/s-0039-1695018

25 Heinke T, Gerharz EW, Bonfig R, Riedmiller H. Ventral onlay urethroplasty using buccal mucosa for complex stricture repair. Urology 2003;61(05):1004-1007http://www.ncbi.nlm.nih.gov/ pubmed/12736024 [Internet]

26 Cohen AJ, Washington S, Butler C, et al. Altruistic donation to improve survey responses: a global randomized trial. BMC Res Notes 2019;12(01):113. Doi: 10.1186/s13104-019-4146-y 


\section{Anexo 1 Encuesta acerca del estado de la urología reconstructiva en Colombia - tendencias en el tratamiento de la estrechez uretral anterior}

\section{ESTADO DE LA UROLOGIA RECONSTRUCTIVA EN COLOMBIA}

Las siguientes preguntas están dirigidas a establecer el estado de la Urología Reconstructiva en nuestra práctica diaria y sólo le tomará cinco minutos. Le agradecemos su valiosa colaboración.

¿En qué rango de edad se encuentra usted?

- 25 - 35 años

- 36 - 50 años

- 51 - 60 años

- Mayor de 60 años

¿Cuántos años lleva de práctica como Especialista en Urología?

- Menos de 5 años

- 5 a 10 años

- 10 a 20 años

- más de 20 años

¿Cuál es su lugar de práctica ?

- Ciudad Capital

- Ciudad Intermedia

¿Maneja usted pacientes con estrechez uretral ?

- Sí

- No

¿Cuántos pacientes con estrechez uretral maneja usted al mes ?

- $1-5$

- $6-10$

- $11-20$

- más de 20

Cuando tiene un paciente con una estrechez de uretra anterior, la conducta más frecuente es:

- Cistoscopia y dilatación

- Uretrotomía interna

- Uretroplastia con injerto

- Remisión a Centro Especializado

Cuando tiene un paciente con una estrechez de uretra posterior, la conducta más frecuente es:

- Uretrotomía Interna

- Uretroplastia perineal

- Uretroplastia con injerto

- Remisión a Centro Especializado

¿Estaría interesado en participar en Foros de Urología Reconstructiva?

- Sí

- No 